\section{Internationalising Linac Research}

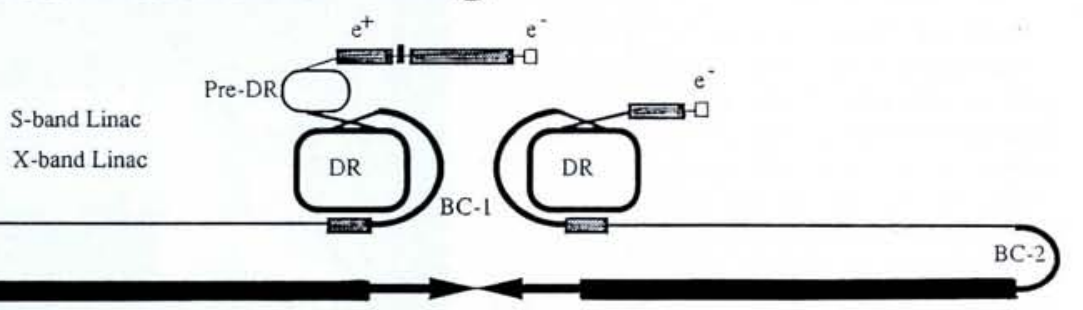

An outline of the KEK (Tsukuba) JLC proposal for a next generation $0.5 \mathrm{TeV}$ linac for particle physics. Electrons are injected into damping rings (DR) and then bunch compressed (BC), pre-accelerated to $10 \mathrm{GeV}$ in an S-band linac, compressed again, and accelerated in two, $7 \mathrm{~km}$ long, $X$-band linacs.

Dr. Burton Richter, Director of the Stanford Linear Accelerator Center, argues that the sensible next step in linear accelerators for particle physics should involve a significantly higher energy than the upgraded LEP machine's $200 \mathrm{GeV}$, as well as aiming for at least twice the top quark's mass (the next physics frontier now estimated to be at $<200 \mathrm{GeV}$ ). So SLAC's Next Linear Collider proposal is for a $500 \mathrm{GeV}$ machine, expandable to $1.5 \mathrm{TeV}$, working in the X-band at four times the present SLAC frequency, representing a trade-off between economies and tolerances. The Japanese JLC proposal by KEK (Tsukuba) envisages similar targets for a $15 \mathrm{~km}$ long, $1 \mathrm{TeV}$ linac collider, which could also be built up in phases starting at $0.5 \mathrm{TeV}$. Based on current linac projects, the cost of the NLC or the JLC would be about \$1 M per $\mathrm{GeV}$, or 500-1500 M\$ in total - significantly less than the SSC.

One alternative is to stay with SLAC's $\approx 3 \mathrm{GHz}$ S-band technology and progressively replace most of the collider with $\mathrm{X}$-band components. Another is to have a high accelerating gradient, entirely superconducting machine working in the $\approx 1.5$ $\mathrm{GHz} \mathrm{L}$-band. But Dr. Richter does not believe in the 25-50 fold decrease in cost which has been claimed in view of less stringent requirements for beam alignment and final focussing (e.g. the TESLA SC-LINAC proposed by an international study group, which could also be constructed in phases, has a beam size of $58 \times 554 \mathrm{~nm}^{2}$ compared with $2.5 \times 220 \mathrm{~nm}^{2}$ for NLC). Meanwhile, the novel $\approx 30 \mathrm{GhZ} \mathrm{K-band} \mathrm{two-beam}$ approach offering high power efficiency (CERN's 2 TeV CLIC proposal calls for 35 $\mathrm{MW} / \mathrm{TeV}$ compared with $200 \mathrm{MW} / \mathrm{TeV}$ for the NLC) is only in the early stages of development at CERN and is seen as the route towards a multi-TeV machine. Another proposal is the DESY (Hamburg) S-band multibranch LC collider using essentially SLAC technology, working at $\approx 3 \mathrm{GHz}$, with an accelerating voltage gradient of $17 \mathrm{MV} / \mathrm{m}$ which is several times smaller than the $80-100 \mathrm{MV} / \mathrm{m}$ used in the other approaches. The LC proposal calls for a $0.5 \mathrm{GeV}$ energy with a five-times smaller luminosity than NLC: the beam size $\left(7.8 \times 192 \mathrm{~nm}^{2}\right)$ remains a problem. Finally, Russian workers have proposed a 1-2 $\mathrm{TeV}$ machine, similar to NLC and JLC but with a larger beam size and a long bunch length.

\section{Joint Projects}

Commenting on internationalisation efforts and whether it was wise to continue is presently $2000 \mathrm{~nm}$ in diameter).

For the injector system, problems asso- ciated with high power sources for a next generation machine based on klystrons augmented by pulse compression units are largely solved, although Russian workers have indicated they would like to collaborate with SLAC on aspects of high power positron sources. KEK may meanwhile take the lead with the M\$10-20 JLC Accelerator Test Facility to design and test an improved damping ring (a sort of storage ring) that would be used to inject a low emittance 1.5-2 GeV beam into each of the two main linacs of a future collider (see figure).

The future is less clear for the development of the main linac accelerator structure, which demands highly accurate beambased alignment, control of beam breakup instability and the solution to problems associated with high gradient acceleration (surface damage, high dark current, etc.). SLAC, KEK, LAL, and INP have collaborated on high gradient experiments for which SLAC has proposed an accelerator test facility along with an engineering model of an NLC section. KEK envisages a similar $100 \mathrm{~m}$ long, $0.5 \mathrm{GeV}$, S-band test linac for its test facility.

There is clearly more-or-less general agreement on what the next major linear collider for particle physics should look like: maybe it will get off the drawing boards as new international projects mature.
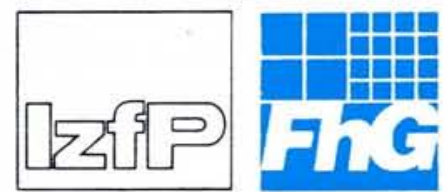 \\ Fraunhofer Institute for Non-Destructive Testing \\ Fraunhofer-Institut für zerstörungsfreie Prüfverfahren (IzfP)
}

The Fraunhofer Institute for Non-Destructive Testing carries out research and development in the field of materials evaluation. We work on ultrasonic, magnetic and $\mathrm{X}$-ray techniques using either state-of-the-art commercial instruments or those based on the results of research work performed in the Institute. The latter are also introduced into the marketplace as prototypes for industrial use, often in close collaboration with commercial licensees.

In the field of acoustic imaging and non-destructive evaluation, we manufacture:

\section{- High frequency ultrasonic inspection equipment}

Our high frequency $(20.200 \mathrm{MHz})$ ultrasonic equipment for inspecting advanced ceramics and composites employs short RF bursts (1 to 99 oscillations at a given frequency) yielding a high signal-to-noise ratio for defect detection but maintaining axial resolution. It can be combined with electronic systems supplied by other manufacturers, either available in a customer's laboratory or purchased separately.

The RF system may also be used for research applications in ultrasonics in solid state physics and materials science, allowing one to obtain back-wall echo sequences in very thin samples $(\geq 100 \mu \mathrm{m}$ depending on the velocity of sound in the material).

\section{- Synthetic aperture focussing (SAFT) equipment}

In SAFT, high frequency data are acquired and stored in a computer to reconstruct defects such as cracks, adhesive failures and inclusions with respect to their location, orientation, shape, and dimensions. A Krautkrämer flaw detector unit USD10 is used with a personal computer for data acquisition and reconstruction. The frequency range covered lies in the range $0.4-20 \mathrm{MHz}$ and the components to be inspected can have either plane or cylindrical surfaces (plates, rods, tubes, etc.) and can be made of steel, aluminium, polymer composites, and ceramics.

\section{- ARGUS phased array system}

The ARGUS phased array system employing 16 ultrasonic channels (or multiples of them) is designed to generate and record ultrasonic signals in the $0.5-8 \mathrm{MHz}$ frequency range. It can be used for defect detection and materials characterization employing both sector and compound scans and VAOK modes.

\section{- Amplitude locus curve (ALOK) equipment}

A low-cost version of an ALOK device, called ALOK-5, has been developed under contract with NDT Electronics, Dudweiler, Germany. ALOK-5 is a modular, compact and fully portable ultrasonic testing system designed for manual and automatic inspections. It includes up to eight channels and a powerful parallel signal processing system. With a noise elimination algorithm and on-line A-scan and C-scan as well as off-line B-scan representations, the device is fully menu-driven and therefore easy to use,

For further information, please contact:

Mr. S. Kraus, Fraunhofer Institute for Non-Destructive Testing,

Bldg. 37, Universität, W-6600 Saarbrücken 11, Germany

Tel.: +49 (681) 30238 11; Fax: + 49 (681) 395 80; Telex: 17681985 IzfPSbr 